\title{
Breathing of heliospheric structures triggered by the solar-cycle activity
}

\author{
K. Scherer and H. J. Fahr \\ Institut für Astrophysik und Extraterrestrische Forschung der Universität Bonn, Auf dem Hügel 71, D-53121 Bonn, Germany
}

Received: 17 September 2002 - Revised: 18 January 2003 - Accepted: 29 January 2003

\begin{abstract}
Solar wind ram pressure variations occuring within the solar activity cycle are communicated to the outer heliosphere as complicated time-variabilities, but repeating its typical form with the activity period of about 11 years. At outer heliospheric regions, the main surviving solar cycle feature is a periodic variation of the solar wind dynamical pressure or momentum flow, as clearly recognized by observations of the VOYAGER-1/2 space probes. This long-periodic variation of the solar wind dynamical pressure is modeled here through application of appropriately timedependent inner boundary conditions within our multifluid code to describe the solar wind - interstellar medium interaction. As we can show, it takes several solar cycles until the heliospheric structures adapt to an average location about which they carry out a periodic breathing, however, lagged in phase with respect to the solar cycle. The dynamically active heliosphere behaves differently from a static heliosphere and especially shows a historic hysteresis in the sense that the shock structures move out to larger distances than explained by the average ram pressure. Obviously, additional energies are pumped into the heliosheath by means of density and pressure waves which are excited. These waves travel outwards through the interface from the termination shock towards the bow shock. Depending on longitude, the heliospheric sheath region memorizes 2-3 (upwind) and up to 67 (downwind) preceding solar activity cycles, i.e. the cycleinduced waves need corresponding travel times for the passage over the heliosheath. Within our multifluid code we also adequately describe the solar cycle variations in the energy distributions of anomalous and galactic cosmic rays, respectively. According to these results the distribution of these high energetic species cannot be correctly described on the basis of the actually prevailing solar wind conditions.
\end{abstract}

Key words. Interplanetary physics (heliopause and solar wind termination; general or miscellaneous) - Space plasma physics (experimental and mathematical techniques)

Correspondence to: K. Scherer

(kscherer@astro.uni-bonn.de)

\section{Introduction to solar cycle - induced variations}

Since Parker (1963) published the first description of the heliospheric interface configuration, it was already obvious that the locations of the solar wind termination shock and the heliopause would vary under different inner heliospheric pressure conditions. However, these early models resulted from stationary equilibrium considerations, requiring that the boundary conditions do not change in time. Model calculations with these restrictions are completed, as soon as an asymptotic state in the locations of the heliospheric structures is reached. Truly dynamical variations of the heliosphere could only be studied in recent years, after refined numerical codes had been developed to model the solar wind - interstellar medium interaction. Following the course of the publications in recent years one can identify a gradual increase in numerical and physical perfections of the models (for a review, see Zank, 1999a). Based on the virtues of these models, studies of time-dependences have meanwhile become feasible.

First studies in this respect showed that the response reaction of the heliospheric shock properties very much depends on the dimensionality in which the problem is described. Barnes (1993, 1994, 1995), Grzedzielski and Lazarus (1993), and Naidu and Barnes (1994) and Wang and Belcher (1998, 1999) have considered the motion of the termination shock in response to a jump in the solar wind density or an interplanetary shock passage, using a one-dimensional planar approximation. Ratkiewicz et al. (1996) then showed that the resulting responses viewed in compression ratios and propagation speeds of the shock, are much less pronounced in a spherically symmetric approach. Similar studies, however, already based on measured perturbations of solar wind properties, were carried out by Whang and Burlaga (1993) and Whang, Lu and Burlaga (1999). These latter authors conclude, that the location of the upstream termination shock is anti-correlated with the sunspot number, i.e. moving inward or outward at the rising or the declining phase of the solar cycle. Since then it is recognized that the heliospheric response to inner heliospheric solar wind variations can only be ade- 
quately described if the whole interaction system is consistently estimated, including all involved time-dependent processes, especially, for example, the back reactions from the heliopause and the bow shock.

In this sense Steinolfson (1994) and Karmesin et al. (1995) have run the first two-dimensional, time-dependent interaction codes to study solar cycle effects and could show that the resulting migrations of the shock location in their timedependent studies turn out to be much more moderate compared to migrations achieved if the shock could instantly react to the actual inner solar wind conditions. This broadened view has then still been substantially improved by Zank (1999b), emphasizing the fact that the interstellar neutral Hatoms, a dynamically important component of the interaction process, was not yet taken into account. By including $\mathrm{H}-$ atoms in a consistent form into a two-dimensional, two-fluid interaction code he showed that the earlier arythmic motion of the termination shock is now reduced as a consequence of a reduction of the upwind to downwind shock layer asymmetry and of the shock distance from the Sun.

Appreciating the relevance played by the $\mathrm{H}$-atom fluid in this context, we now feel strongly encouraged to revisit the problem of heliospheric interface responses to solar cycle variations, however, including now, in addition to the approach presented by Zank (1999b), three more fluid components of dynamical relevance within this context. These latter components are pick-up ions (PUIs), anomalous cosmic rays (ACRs) and galactic cosmic rays (GCRs) (see Fahr et al., 2000; Scherer et al., 2002).

\section{The solar-cyclic response of the 5-fluid heliosheath}

The theoretical basics of the 5-fluid model has already been published by Kausch (1998), Fahr et al. (2000) and Fahr (2000). Thus, we do not want to describe all the physical and numerical details here again. We only briefly summarize that besides protons of solar and interstellar origin, we consistently include interstellar $\mathrm{H}$-atoms, heliospheric $\mathrm{H}$ pick-up ions, H-ACRs and GCRs as separate fluids which are dynamically and thermodynamically coupled to build the multifluid interaction system. PUIs are co-moving with the local solar wind, but behave thermodynamically as an independent fluid with separate values of density, temperature, sound speed and pressure. ACRs are injected at the shock with a rate dependent on the local strength of the shock into the Fermi- 1 acceleration process. This rate corresponds to a specific fraction of the PUI flux which locally passes over the shock. This injection constitutes a local ACR source at the shock which is taken into account in an energy-averaged ACR transport equation describing convection and spatial diffusion of this high-energy $10 \mathrm{MeV} /$ nuc fluid. Finally, we include the GCRs by coupling to the other fluids an energyaveraged GCR transport equation. Hereby, the coupling occurs due to convective GCR changes connected with the local plasma bulk flow, and due to a modulation of the plasma motion by ACR and GCR pressure gradients.
Table 1. The parameter for the calculations, while the interstellar parameters are assumed to be valid at "infinity"

\begin{tabular}{lll}
\hline \multicolumn{3}{c}{ Solar wind and other parameters at the Earth's orbit } \\
\hline bulk velocity & $v_{s w}$ & $400 \mathrm{~km} / \mathrm{s}$ \\
proton number density & $n_{s w}$ & $5 \mathrm{~cm}^{-3}$ \\
proton temperature & $T_{s w}$ & $100000 \mathrm{~K}$ \\
period of solar cycle & $P$ & 11 years \\
Diffusion coefficient of GCR & $\kappa$ & $1 \cdot 10^{22} \mathrm{~cm}^{2} / \mathrm{s}$ \\
Rigidity of GCR and ACR & $R$ & $1 \mathrm{MV}$ \\
\hline
\end{tabular}

Parameters of the local interstellar medium

\begin{tabular}{lll}
\hline bulk velocity of hydrogen & $v_{H_{L I S M}}$ & $26 \mathrm{~km} / \mathrm{s}$ \\
number density of hydrogen & $n_{H_{L I S M}}$ & $0.1 \mathrm{~cm}^{-3}$ \\
hydrogen temperature & $T_{H_{L I S M}}$ & $8000 \mathrm{~K}$ \\
bulk velocity of protons & $v_{H_{L I S M}}$ & $26 \mathrm{~km} / \mathrm{s}$ \\
number density of protons & $n_{H_{L I S M}}$ & $0.1 \mathrm{~cm}^{-3}$ \\
proton temperature & $T_{H_{L I S M}}$ & $8000 \mathrm{~K}$ \\
\hline \multicolumn{2}{c}{ Parameter of energetic particles in the LISM } \\
\hline GCR energy density & $e_{G C R}$ & $0.28 \mathrm{eV} \mathrm{cm}{ }^{-3}$ \\
mean kinetic energy of GCR & $E_{k i n_{G C R}}$ & $16 \mathrm{GeV}$ \\
Diffusion coefficient & $\kappa$ & $3.9 \cdot 10^{28} \mathrm{~cm}^{2} / \mathrm{s}$ \\
ratio between perpendicular & $\kappa_{\perp} / \kappa \|$ & 0.03 \\
to parallel diffusion & & \\
mean kinetic energy of ACRs & $E_{k i n_{A C R}}$ & $514 \mathrm{MeV}$ \\
injection coeffiecient of ACR & $\alpha_{A C R}$ & 2.5
\end{tabular}

The parameters for a stationary heliosphere are shown in Table 1 . These model calculations, after reaching its asymptotical state, are used as input for modeling the solar cycle variations. All different solar cycle models discussed below use this stationary state as a starting point for the new calculation.

The high-energy components, i.e. the ACRs and the GCRs, also represent two additional fluid species diffusing in space due to being scattered at MHD-wave turbulences. These species, in a mathematical sense, enforce the upgrading of the system of coupled partial differential equations to a system of second order equations. The inclusion of PUI-, ACR- and GCR-fluids in a physical sense opens up new reaction modes for the whole interaction system, if timedependent inner boundary conditions modeling typical solar cycle variations are installed.

As is well known, such variations have been reported on the basis of deep space probe data obtained with PIONEER and VOYAGER spacecraft by Kayser, Barnes and Mihalov (1984) and Lazarus and McNutt (1990). They essentially show cycle-periodic variations of the solar wind mass flow and dynamical pressure roughly by a factor of 2 , with maxima delayed by roughly 4 years with respect to the activity cycle maximum. As can be extracted from data presented by Lazarus and Belcher (1987), Gazis (1994) or McComas et al. (2000) this solar-cyclic variation of the solar wind dynamical pressure is connected with a nearly constant solar 


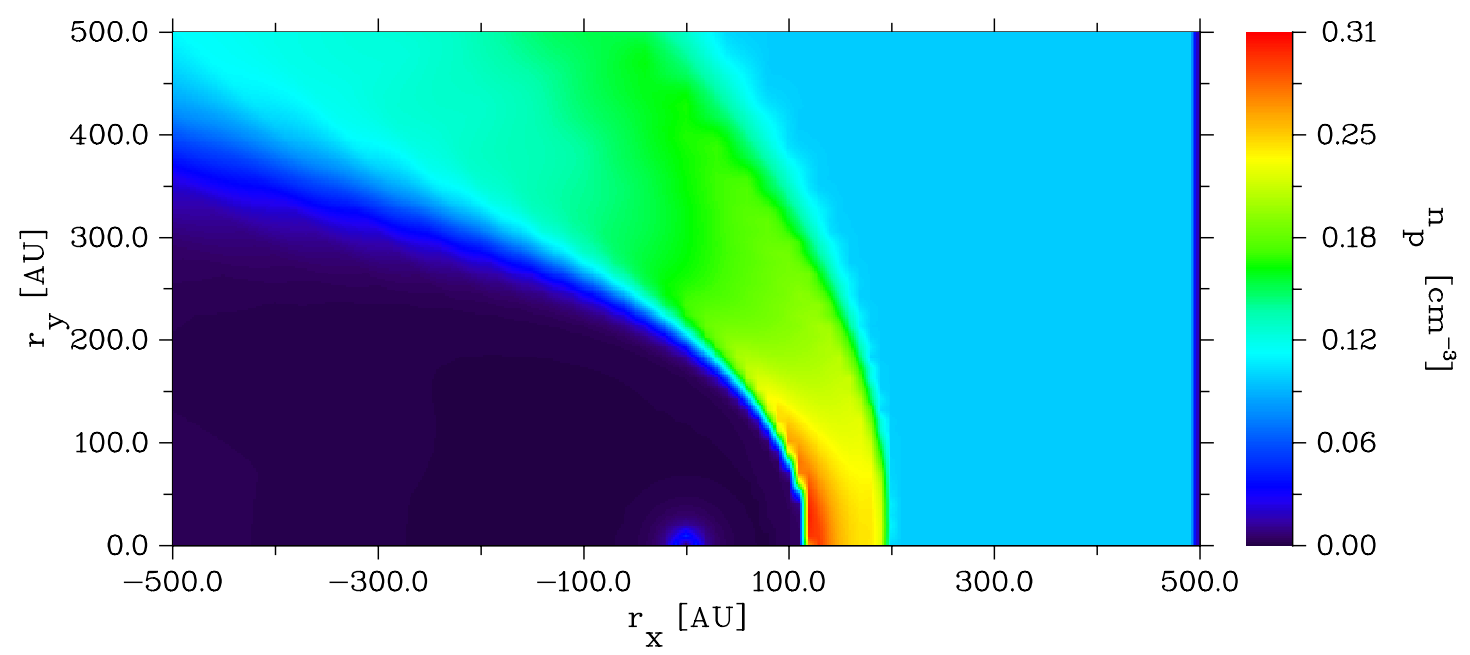

(a) static heliosphere

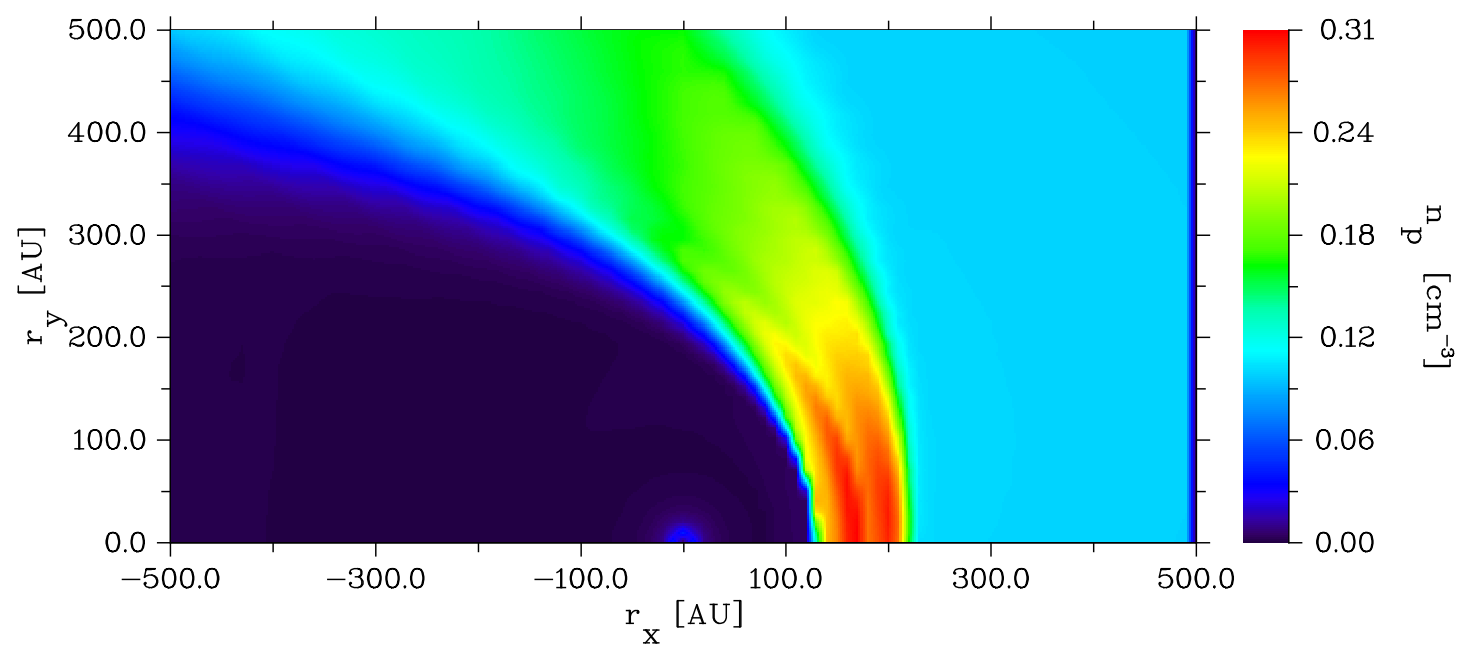

(b) dynamic heliosphere

Fig. 1. The proton density variations for the static and the dynamic heliosphere (LISM flow from the right). In the upper panel the static model based on the parameters given in Table 1 is shown, while in the lower panel the dynamic heliosphere is presented, in which the solar wind speed and density at the inner boundary varies according to a sinusoidal law between $300 \mathrm{~km} / \mathrm{s}$ and $500 \mathrm{~km} / \mathrm{s}$. For the varying distances of the termination shock and the bow shock see Fig. 4.

wind mass flux, since solar wind velocity and density appear to be appropriately anti-correlated. In view of these findings we prefer here models in which the mass flow is constant during the solar cycle, as described in the next section.

\section{Solar cycle models}

At a distance of $5 \mathrm{AU}$, taken as the inner boundary of our simulation model, the radially symmetric inner solar wind velocity becomes time-dependent, with velocity magnitudes varying periodically with the solar cycle. The solar wind mass flow, e.g. $\rho_{s w} v_{s w}$, thereby is kept constant, meaning that the density $\rho_{s w}$ is anti-correlated with the velocity $v_{s w}$. This also means that the velocity varies by the same factor, as does the ram pressure $\rho_{s w} v_{s w}^{2}$. We modeled two types of solar cycle activity: (1) a pure sinusoidal variation and (2) a more complicated but perhaps a more realistic one, with an exponentially modulated amplitude, given by Fahr et al. (1987) and Fahr and Scherer (1990):

$v_{s w}(t)=v_{s w_{0}}+\Delta v_{s w} \cos \left(\omega_{s} t+\phi\right) \exp \left(\cos \left(\omega_{s} t+\phi\right)\right),(1)$ where $\omega_{s}$ is the solar cycle frequency. $v_{s w 0} \cong 359 \mathrm{~km} / \mathrm{s}$ and $\Delta v_{s w} \cong 162 \mathrm{~km} / \mathrm{s}$ are chosen in such a way that the solar wind velocity during maximum conditions reaches $v_{\max }=$ $800 \mathrm{~km} / \mathrm{s}$ while during minimum conditions it is $v_{\min }=$ 


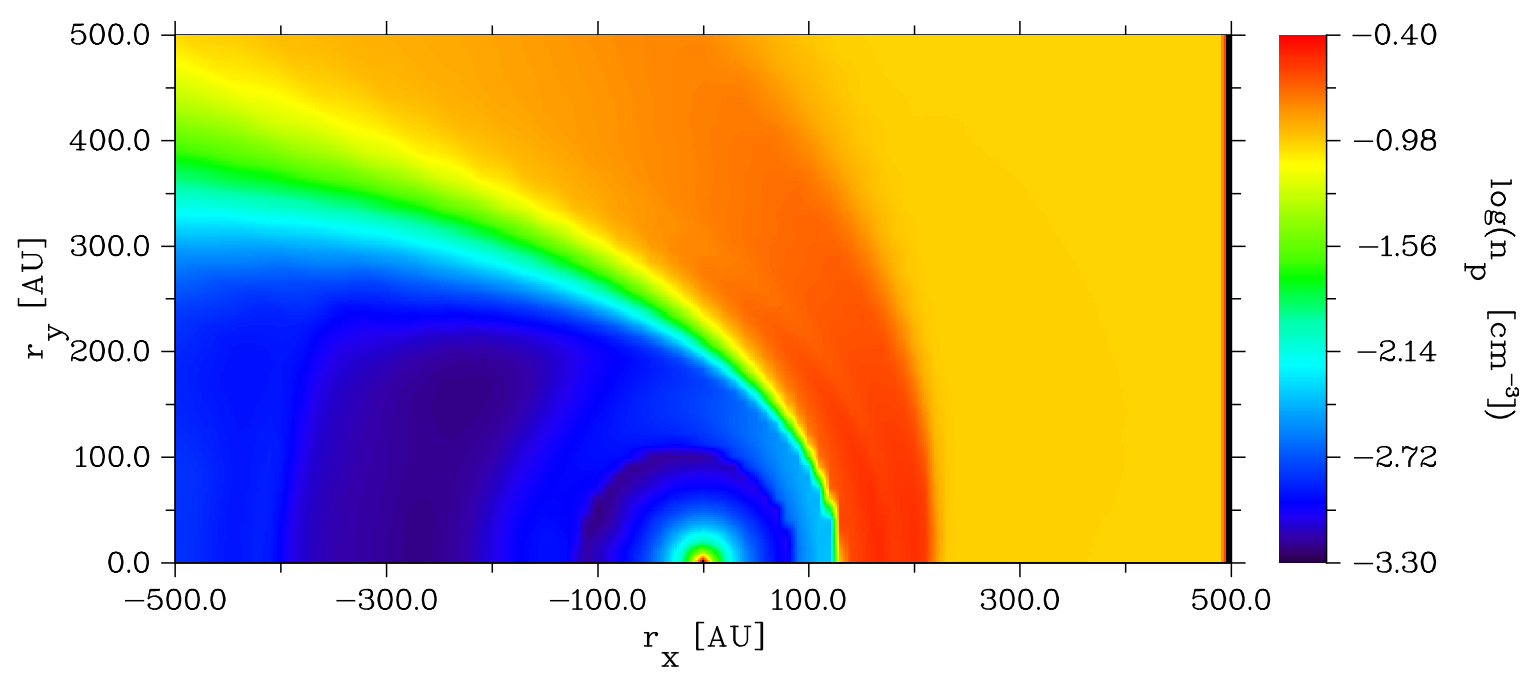

Fig. 2. Same as in Fig. 1 in a logarithmic scale, which enhances the effects in heliotail region.

$300 \mathrm{~km} / \mathrm{s}$. The phase $\phi$ is needed to adapt to a stationary heliosphere with a solar wind speed of $v_{s} w_{\text {stationary }}=400 \mathrm{~km} / \mathrm{s}$.

We also used a sinusoidal law to model the solar cycle variations:

$v_{s w}=v_{s w_{0}}+\Delta v_{s w} \sin \left(\omega_{s} t+\phi\right)$,

where the parameters $v_{s w_{0}}, \Delta v_{s w}, \omega_{s}$, and $\phi$ have the same meaning as in Eq. (1).

In the preceding models in the literature, the authors varied either the solar wind speed or the solar wind density. In our models we kept the solar mass flux constant, requiring the variability of both the solar wind speed and the solar wind density simultaneously. Therefore, the associated solar wind density at the inner boundary is adopted such that the resulting mass flux at $5 \mathrm{AU}$ is constant during the course of the solar cycle and is equal to $\Phi_{m 0}(5 \mathrm{AU})=$ $v_{s w} \cdot n_{s w}(5 \mathrm{AU}) \mathrm{m}_{p}=8 \cdot 10^{6} \mathrm{~m}_{p} \mathrm{~cm}^{2} \mathrm{~s}^{-1}$, which is approximately the value measured at the Ulysses spacecraft $\Phi_{m 0, U}=1.2 \cdot 10^{7} \mathrm{~m}_{p} \mathrm{~cm}^{2} / \mathrm{s}^{-1}$ (McComas et al., 2000).

\section{Snapshots of the large-scale heliospheric structure}

In Figs. 1 to 3 the proton and hydrogen density of the heliosphere are shown in the form of color-coded plots. In the following displays we keep the set of the static heliosphere parameters as standard and only vary in time the solar wind speed $v_{s w}=v_{s w}(t)$ as discussed above, according to Eqs.(1) and (2), where the solar mass flux is constant and hence, the solar wind number density $n_{s w}=n_{s w}(t)$ is anti-correlated to the solar wind speed. The period of the solar cycle is kept constant and is adopted with 11 years.

For a pure sinusoidal solar-cyclic variation of the solar wind speed between $V_{s w_{\min }}=300 \mathrm{~km} / \mathrm{s}$ and $v_{s w_{\max }}=$ $500 \mathrm{~km} / \mathrm{s}$, density contours for protons at a specific phase of the solar cycle (approximately in the middle of the cycle) are shown in Figs. 1b, 2 and 3b (i.e. a phase snapshot of the dynamic heliosphere). As one can easily identify by eye, the main structure features of the dynamic heliosphere appear to be a little blown up compared to those of the static heliosphere, both when viewed in proton and hydrogen densities. Furthermore, in the dynamic heliosphere one can clearly see (Fig. 1b) two proton density waves propagating outwards from the heliopause towards the bow shock. The waves traveling outwards, as can be controlled in a cinematographic sequence of successive heliospheric states, clearly are pointed out as subsonic waves running into the bow shock. This most likely should also have implications for a post-terminationshock acceleration of ACRs and for a modulation of GCRs and ACRs downstream of the termination shock.

Since the proton density in this contour plot varies over several orders of magnitude, we present in addition to the linear scale a logarithmic scale (see Fig. 2), to enable the visibility of all the wavy features, especially in the heliotail, which can be observed in the inner and the outer heliosphere, as found in our models. An animation of these features can also be found under http://www.aef-ev.de/Kom03/heliopuls.

Similar features can be observed for the hydrogen density, as presented in Fig. 3 within a logarithmic scale, to better pronounce the details in the heliotail. Moreover, to enhance the effects we allow for a maximal solar wind speed of $800 \mathrm{~km} / \mathrm{s}$. The waves in the hydrogen wall cannot be resolved in this scale, but the "chaotic" behavior of the hydrogen distribution inside the heliosphere is clearly seen.

Alternatively, we also studied the heliospheric solar cycle variations resulting from the time-dependence given by Eq. (1), which seems to be more realistic and better confirmed by data showing that, after an extended quiet period, the solar wind speed then rapidly increases towards its maximum and then again rapidly decreases from this maximum towards a new extended minimum. These exponential features, rather than a sinusoidal triggering, resemble more the 


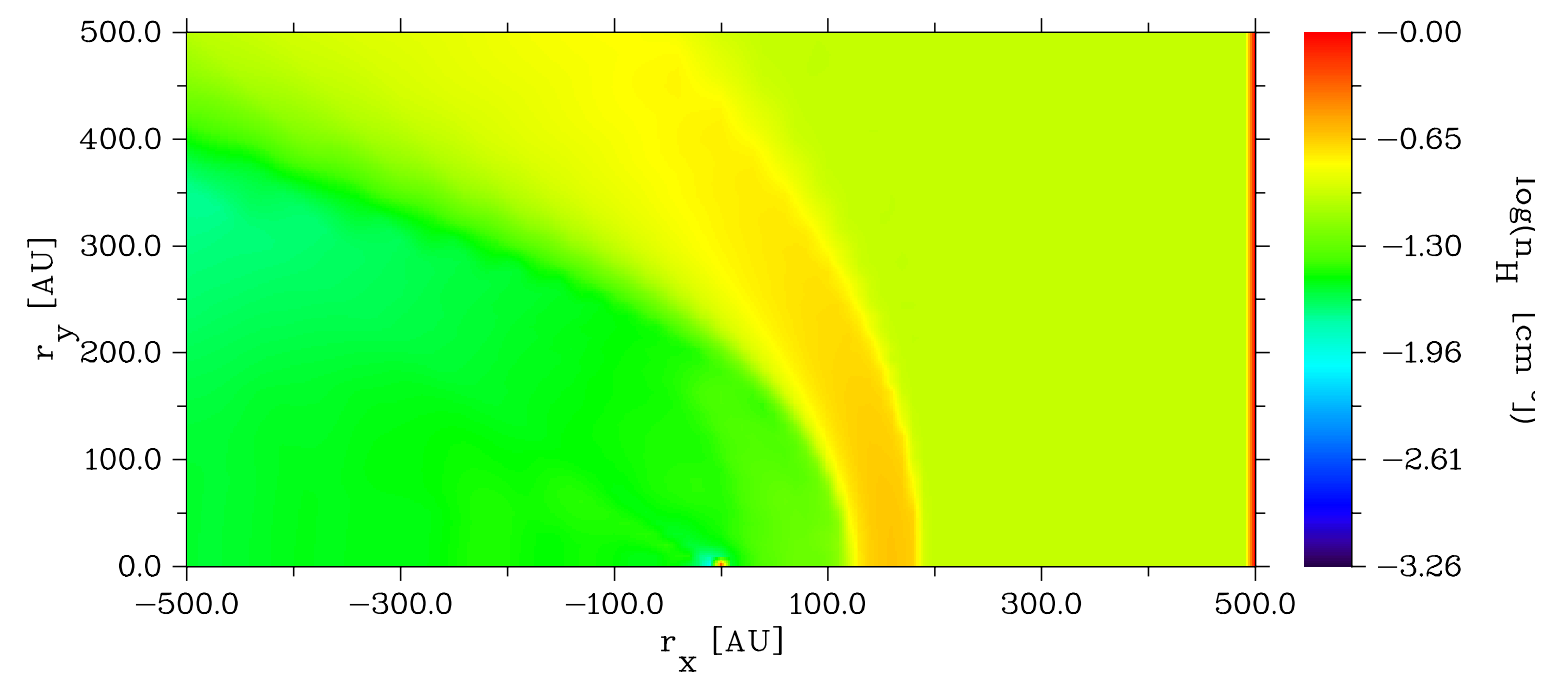

(a) static heliosphere

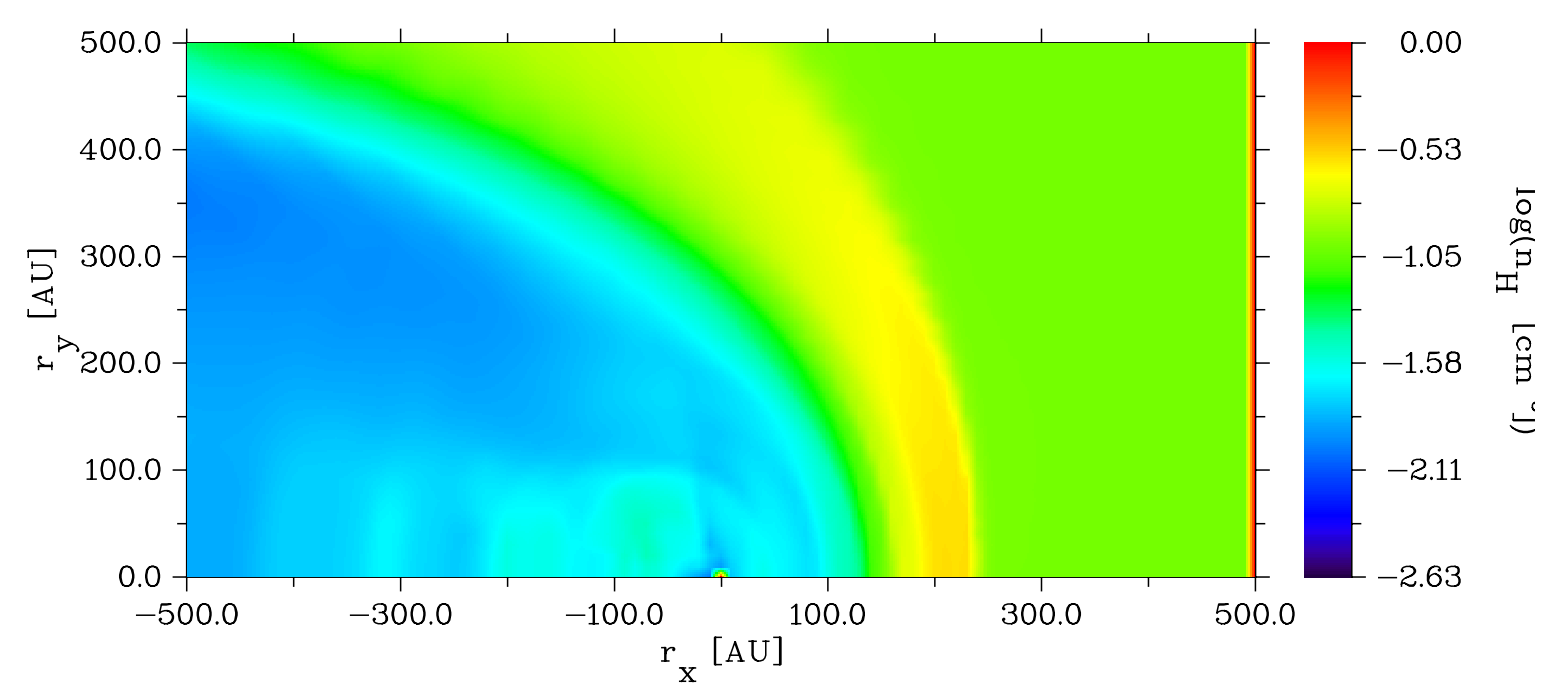

(b) dynamic heliosphere

Fig. 3. Same as in Fig. 1 for the hydrogen distribution. To enhance the effects the solar wind speed variations reach from $300 \mathrm{~km} / \mathrm{s}$ to $800 \mathrm{~km} / \mathrm{s}$ for a pure sinusoidal law. Moreover, a logarithmic scale is chosen.

case of a large Global Merging Interaction Region (GMIR) running into the termination shock. We have modeled solar wind speed variations between $v_{s w_{\text {min }}}=300 \mathrm{~km} / \mathrm{s}$ and $v_{s w_{\max }}=500 \mathrm{~km} / \mathrm{s}$ or $v_{s w_{\max }}=800 \mathrm{~km} / \mathrm{s}$ for both solar cycle models.

\section{Distances to termination and bow shock in different solar cycle models}

In Fig. 4 five different representations of solar cycle variations are used by us to model the solar-cyclic heliosphere variation. The differences in the results can then be com- pared. The minimum solar wind speed $v_{s w_{\min }}=300 \mathrm{~km} / \mathrm{s}$ is kept fixed in all of the five models. The black line represents a sinusoidal modeling with a maximal solar speed of $v_{s w_{\max }}=500 \mathrm{~km} / \mathrm{s}$. In this model no PUIs, ACRs, and GCRs are taken into account, i.e. their dynamical role and relevance is suppressed (model 1). In contrast, in the other four models all five energetic particle species (i.e. protons, hydrogen atoms, PUIs, ACRs, GCRs) are consistently taken into account with their mutual dynamical interactions. While the red curve (model 2) and green curve (model 3) show variations according to Eq. (1), with a maximal solar wind speed of $v_{s w_{\max }}=500 \mathrm{~km} / \mathrm{s}$ and $v_{s w_{\max }}=800 \mathrm{~km} / \mathrm{s}$, respectively, the blue curve (model 4) and cyan curve (model 5) show re- 


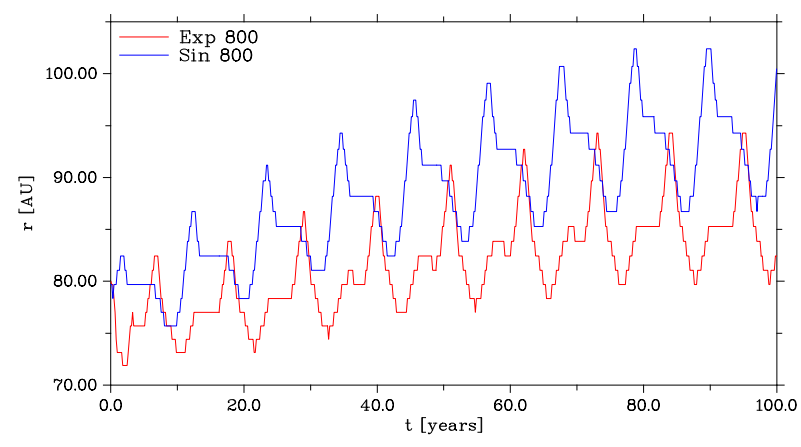

(a) Variation of the TS distance

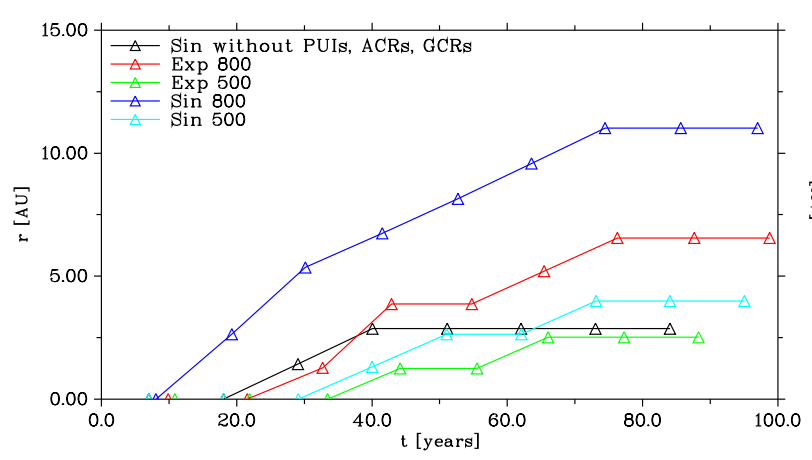

(c) Mimimal TS differnce

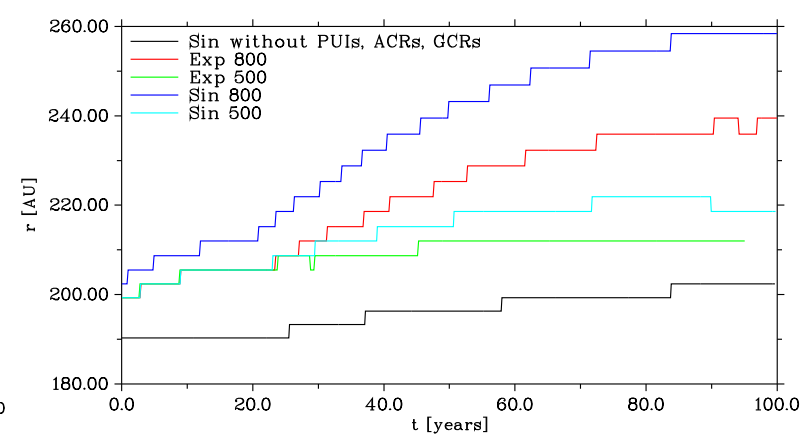

(b) Variation of the BS distance

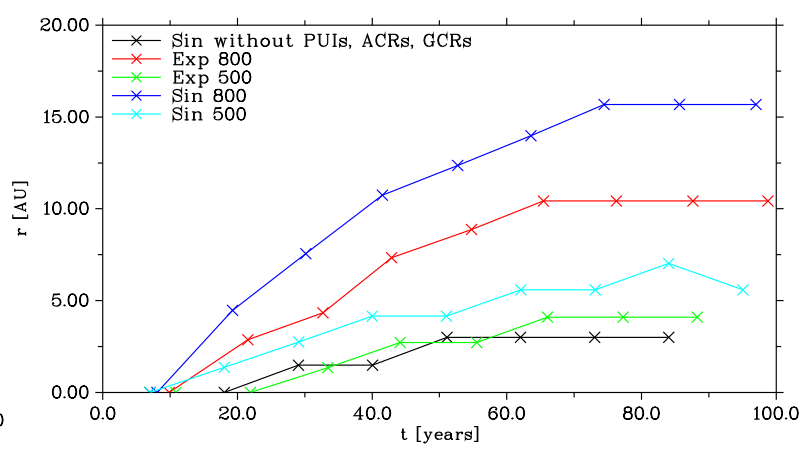

(d) Mimimal BS difference

Fig. 4. For five different solar cycle models the variability of the termination shock and bow shock is shown (see text for details). The relative minimal and maximal distance between the terminations shock of a static and dynamical heliosphere during the minimum and maximum phase, respectively, are plotted in the lower panel.

sults for an analogous amplitude of the solar wind speed, but now for a sinusoidal variation.

In the case of model 1 (in which only hydrogen and protons are taken into account), we used a similar parameter set, as discussed in Table 1, but without any interaction of the PUIs, ACRs, and GCRs as input for the sinusoidal solar cycle model $\left(v_{s w_{\max }}=500 \mathrm{~km} / \mathrm{s}\right)$. It turns out that the distance of the termination shock is larger than compared to the standard stationary model where all 5 -fluid components are used. Furthermore, the TS in model 1 migrates only a small distance outwards and then more or less purely oscillates around its average position. After almost four solar cycles the minimum termination shock distance (Fig. 4c) is constant for the following cycles, which also holds true for the bow shock. In contrast, the bow shock distance for models $2-5$ is still slightly growing after 10 solar cycles.

The phase lag between the sinusoidal and exponential models (Fig. 4a) is caused by the condition that they have to fit to the stationary model at $t=0$. While the amplitudes are largest for the models with $v_{s w_{\max }}=800 \mathrm{~km} / \mathrm{s}$, the form of the curves is much different: while the motion of the TS in the exponential model shows slow growth, with a flat saddle in the slope, the sinusoidal models increase fast and mono- tonically towards their maximal position. The decrease towards the minimum condition of the TS is slow and (almost) monotonic for the sinusoidal models, while the exponential models decrease rapidly and monotonically towards the minimum position. In all four models an increasing in the minimal and maximal distance of the TS can be observed. This is shown in detail in Fig. $4 \mathrm{c}$ for the relative minimal distance of the termination shock and in Fig. $4 \mathrm{~d}$ for its relative maximal distance. The relative distances are calculated as the respective deviations from the static model.

Depending on the amplitude of the solar wind speed variation in five of the solar cycle models, the distance of the bow shock migrates without any oscillation. While no equilibrium location seems to be achieved in model 1 , for models $2-5$ an equilibrium position is reached almost after 6-8 solar cycles. It should be mentioned here that the bow shock has a nearly stable location over one solar cycle. The jumps are occuring due to numerical uncertainties and the handling of large data sets. Only the tendency of a systematic increase in the bow shock distance over several consecutive cycles expresses a physically relevant fact.

From the above mentioned, it becomes clear that the heliosphere has a memory and recognizes at least a couple of solar 


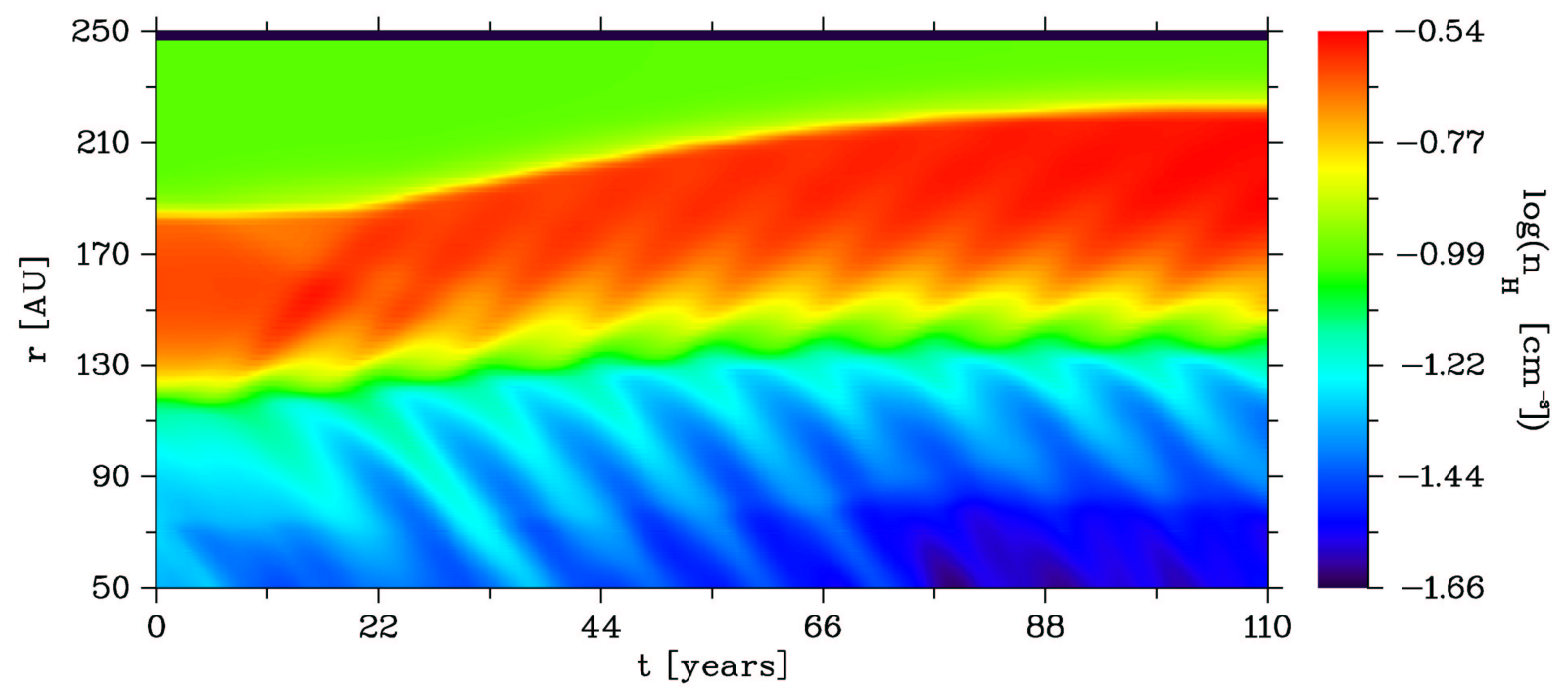

Fig. 5. The variation of the hydrogen density along the stagnation axis for almost 11 solar cycles.

cycles. Thus, after an extended period of low solar activity, as was the case in the Maunder Minimum (1645-1715, where almost no solar cycle activity was observed, e.g. Beer 2000), some time is needed for the termination shock and bow shock to reach well adapted positions. The behavior of and the importance for the cosmic ray fluxes in the outer heliosphere will be discussed elsewhere by Scherer et al. (2003).

In the following, we will concentrate on model 5, i.e. the exponential law with a solar wind speed variation from $300 \mathrm{~km} / \mathrm{s}$ to $800 \mathrm{~km} / \mathrm{s}$, because it shows the most pronounced features. All what will be discussed below holds true for the other models $2-4$.

Instead of showing a snapshot in time, as in Figs. 1-2, we now show in Figs. 5-8 an isochronic cut through space, i.e. along one direction (in this paper we chose the stagnation line), as a function of time. There, it is nicely seen that the maximum distances to the termination shock, the heliopause and the bow shock systematically increase for about 6 solar cycles. Only after about that time do these structure features purely oscillate in distances with the solar cycle.

\section{Hydrogen waves}

In Fig. 5 the hydrogen density variation is shown, while the corresponding features for the proton density are discussed in Scherer and Fahr (2002). As expected. the region ahead of the heliopause appears as a region of enhanced $\mathrm{H}$ atom densities. However, in the dynamic heliosphere the socalled "hydrogen-wall"-structure turns out to be a substantial time variable feature. The changes in the formation of the wall configuration are associated with $\mathrm{H}$-atom density waves propagating inwards and outwards from this wall, with about equal velocities of the order of $25 \mathrm{~km} / \mathrm{s}$. In addition, it can be recognized that the thickness (extent) of the hydrogen wall is periodically varying according to the frequency of the solar cycle. This very interesting phenomenon could be directly controlled by observation of hydrogen-wall modulated absorption features in stellar hydrogen spectra near H-Lyman- $\alpha$ (see Linsky and Wood, 1996; Linsky et al., 1993), because the spectral imprints to these spectra from the hydrogen wall should periodically vary over the solar cycle.

But not only can heliospheric hydrogen be detected by observing Lyman- $\alpha$ line features. In addition, inconsistencies in the Lyman- $\alpha$ spectra intepretations from other stars are also explained as resulting from their astrospheres, i.e. as their "stellar heliospheres" (Wood et al., 2000). Thus, stellar cycle induced astrophere changes are also reflected in the variations of their hydrogen wall, and the observation of the resulting Lyman- $\alpha$ emission will allow for an indirect observation of time-varying stellar winds around Sun-like stars.

\section{Shock waves and compressible waves}

To study the characteristic features of the waves traveling towards the bow shock, we estimated the proton Mach-number. In Fig. 6 it is plotted along the stagnation line for almost 11 solar cycles. The heliopause is recognized here as the black (dark blue) area between $100 \mathrm{AU}$ and $139 \mathrm{AU}$. It can also be seen that subsonic regions extend towards the termination shock, as well as towards the bow shock. But, nevertheless, large areas in the heliosheath are filled with traveling subsonic waves.

The radial velocities of the protons between $100 \mathrm{AU}$ and $180 \mathrm{AU}$ (Fig. 7) also show a systematic change from a small inflow to a small outflow of material between the heliopause and the bow shock. This should provide an increase in the MHD-turbulence. Its estimation must be done elsewhere with a different model as the 5-fluid approach. Nevertheless, from our model we can presently conclude, that the periodic increase and decrease in the Mach-number, as well as in the 


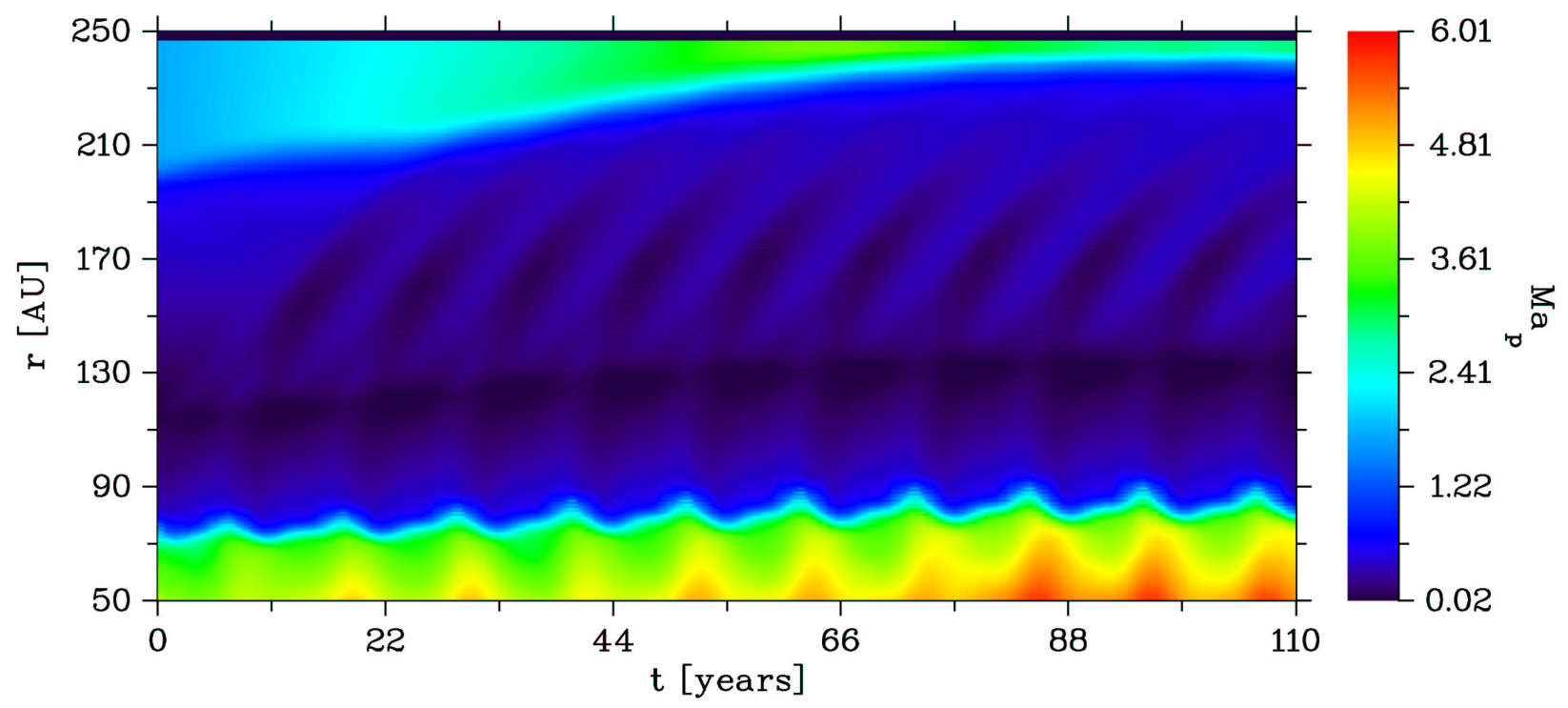

Fig. 6. The Mach-number of the protons along the stagnation axis for almost 11 solar cycles.

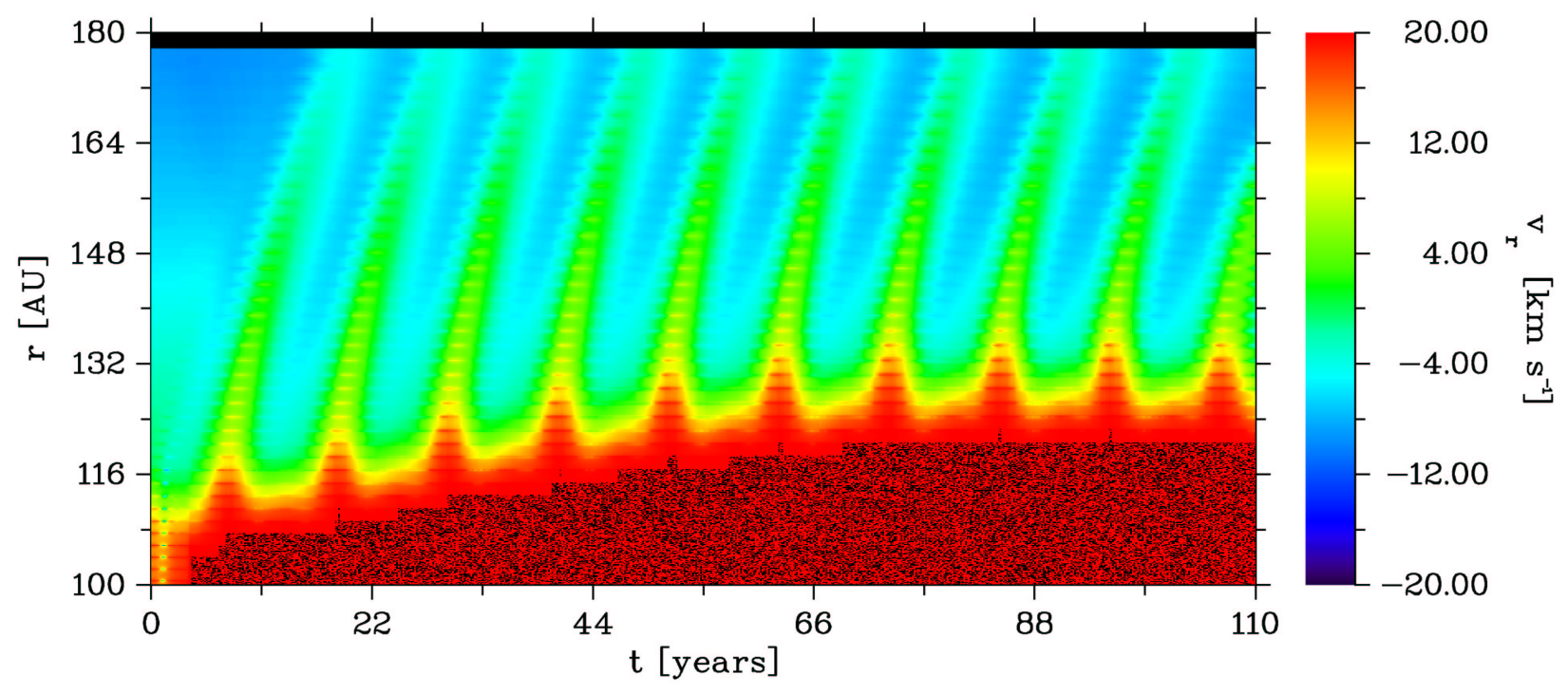

Fig. 7. The variation of the radial proton velocity in the sheath between termination shock and bow shock

proton velocities, causes similar increases and decreases in the turbulence levels. The latter affects the transport of energetic particles, as well as the effectiveness of the acceleration of ACRs. Based on our model data presented here, Scherer et al. (2003) discuss some of the consequences for GCRs.

\section{Merging waves in the tail region}

Figure 8 also shows a time versus distance plot for the proton density in the heliotail. Here, the densities are very small, but, nevertheless, the solar activity cycles blow even more "holes" into the density patterns. These depression areas start to expand and then propagate further out where they slowly decay, i.e. they induce proton density increases at larger distances. Furthermore, density waves rising from different solar cycles merge at even larger distances, which also should increase the MHD-turbulence levels. These merging waves may accelerate newly ionized particles, which are created by charge exchange processes between the protons and hydrogen atoms in the heliotail, to some $\mathrm{keV}$ and which can be observed on SOHO (Czechowski et al., 2001).

The merging wave regions are moving toward the termination shock, but did not reach it after 10 solar cycles. Therefore, we will have to perform longer runs of our models to better study the long-term behavior in the tail. 


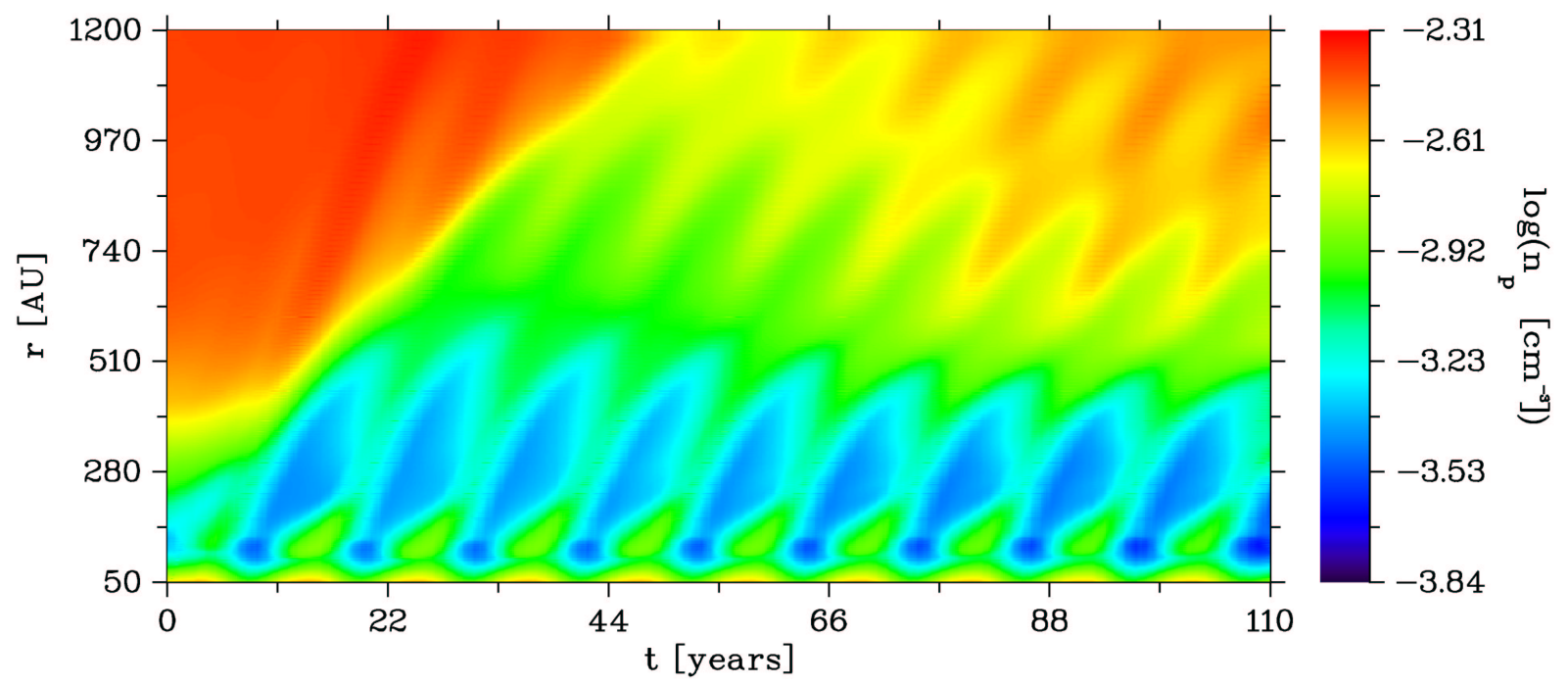

Fig. 8. Variation of the proton density in the tail. The density waves are merging in the outer regions of the outer tail. In the merging interaction regions the production of high energetic particles (to some keV) should be possible.

\section{More dimensions needed?}

The heliosphere is an essentially three-dimensional structure, not only due to the frozen-in magnetic fields carried by the solar wind, but also due to the three-dimensional asymmetric distribution of the solar wind momentum flow. The effects of the three-dimensional distribution of the solar wind momentum flow during solar minimum may be handled by separate static axisymmetric models, under the assumption that as observed by Ulysses (McComas et al., 2000), there exists a sharp transition region between the slow and fast solar wind. But the principle physical effects of the fast solar wind and the slow ecliptical solar wind can only be studied by dynamically fully developed 3-D models, because the transport of the cosmic rays depends on the different turbulence levels caused by the three-dimensional structure of the frozen-in magnetic field as well as that of the 3-D solar wind momentum distribution. Hence, the solar cycle dependence studied here is a first attempt to understand the difference between static and dynamical models in a 2-D approximation for different ecliptical boundary conditions simulating an ecliptic cut through the three-dimensional structure of the heliosphere. Thus, the models $2-4$, discussed above, give a first glimpse of the nature of the three-dimensional heliosphere.

On the other hand, the three-dimensional magnetic field induces different polarities in both hemispheres, which can be observed in the modulation of cosmic rays (e.g. Potgieter, 1998; Fichtner, 2001). These effects cannot be modeled in our present five-fluid code. Nevertheless, axisymmetric models, with a kinematic treatment of the magnetic field to calculate the diffusion coefficients, describe the CR-transport in the ecliptic quite well. Therefore, the modulation of CRs in our model is a first attempt to include these important species in heliospheric models.
In addition to the above discussed three-dimensional structure of the heliosphere from the inside, an outside LISM magnetic field may have to be expected, giving an imprint on the heliospheric configuration (Ratkiewicz et al., 1996). At this time, no three-dimensional models exist which include the frozen-in solar wind magnetic field as well as the magnetic field of the LISM, nor do they handle sophisticated cosmic ray transport models. Thus, our present five-fluid code can be understood as a first step in the development of more sophisticated heliospheric models.

\section{Summary}

We presented time-dependent models for the large-scale heliosphere, in which the solar cycle dependence was described by a periodic variation of the solar wind speed and density, respectively. Keeping the solar wind mass flow constant leads to moderate variations, which causes an overall migration of the termination shock, as well as the bow shock to larger distances.

In the heliosheath region solar cycle induced plasma waves and waves of neutral atoms (i.e. hydrogen) are merging into the bow shock. These waves affect the propagation of cosmic rays, as well as the acceleration of its anomalous component.

It turns out that periodic variations of the solar wind change the structure of the heliosphere remarkably. For the first time we could model the "heliospheric memory" (see, also, Scherer and Fahr, 2002), i.e. the fact that not only the last solar cycle influences the structure of the outer heliosphere but up to 6-7 previous cycles (and even more in the tail region) must be taken into account to correctly model the dynamic heliosphere and its hysteresis effects. Because the large-scale structure and physics of the heliosphere is used in related fields, such as the propagation of cosmic rays, 
production of energetic neutral atoms, the interpretation of Lyman- $\alpha$ line from other stars, etc., the dynamical heliosphere should be taken into account in future applications.

Acknowledgement. The authors are grateful for financial support granted to them by the Deutsche Forschungsgemeinschaft in the frame of the project "Heliotrigger" (Fa 97/28-1).

Topical Editor R. Forsyth thanks two referees for their help in evaluating this paper.

\section{References}

Barnes, A.: Motion of the heliospheric termination shock: A gasdynamic model, J. Geophys. Res., 98, 15 137, 1993.

Barnes, A.: Motion of the heliospheric termination shock, 2, Energy loss due to energetic particle acceleration, J. Geophys. Res., 99, 6553, 1994.

Barnes, A.: Motion of the heliospheric termination shock at high heliospheric latitude, Space Sci. Rev., 72, 233, 1995.

Beer, J.: Neutral monitor records in broader historical context, Space Sci. Rev. 93, 107-119, 2000.

Czechowski, A., Fichtner, H., Grzedzielski, S., Hilchenbach, M., Hsieh, K. C., Jokipii, J. R., Kausch, T., Kota, J. and Shaw, A.: Anomalous cosmic rays and the generation of energetic neutrals in the region beyond the termination shock, Astron. Astrophys., $368,622,2001$.

Fahr, H. J.: The Multifluid Character of the 'Baranov' Interface, Astrophys. Space Sci., 274, 35-54, 2000.

Fahr, H. J. and Lay, G.: Remote diagnostic of the heliospheric termination shock using neutralized post shock pick-up ions as messengers, Astron. Astrophys., 356, 327-334, 2000.

Fahr, H. J. and Scherer, K.: Heliospheric neutral gases in threedimensional and time-dependent solar force and ionization fields, Astron. Astrophys., 232, 556-564, 1990.

Fahr, H. J., Kausch, T., and Scherer, H.: A 5-fluid hydrodynamic approach to model the solar system-interstellar medium interaction, Astron. Astrophys., 357, 268-282, 2000.

Fahr, H. J., Nass, H. U., and Rucinski, D.: Modelling of the interplanetary helium EUV-resonance glow for time-dependent solar radiation conditions, Ann. Geophysicae, 5, 255-263, 1987.

Fichtner, H.: Anomalous Cosmic Rays: Messenger from the Outer Heliosphere, Space Sci. Rev., 95, 639-754, 2001.

Gazis, P. R.: Pioneer and Voyager observations of solar cycle variations in the outer heliosphere, Geophys. Res. Lett., 21, 17431746, 1994.

Gruntman, M., Roelof, E. C., Mitchell, D. G., Fahr, H. J., Funsten, H. O., and McComas, D. J.: Energetic neutral atom imaging of the heliospheric boundary region, J. Geophys. Res., 106, $15767-$ $15781,2001$.

Grzedzielski, S. and Lazarus, A. J.: 2- to 3-kHz continuum emissions as possible indications of global heliospheric "breathing", Space Sci. Rev., 98, 5551-5558, 1993.

Izmodenov, V., Gruntman, M., Baranov, V. B., and Fahr, H. J.: Heliospheric ENA fluxes: How Sensitive Are They to the Ionization State of LIC?, Space Sci. Rev., 97, 413-417, 2001.

Karmesin, S. R., Liewer, P. C., and Brackbill, J. U.: Twodimensional simulations of the motion of the termination shock in response to 11 years variation in the solar wind, Geophys. Res. Lett., 22, 1153-1156, 1995.

Kausch, T.: Ein hydrodynamisches Mehrkomponentenmodell zur Beschreibung der Wechselwirkung zwischen Heliosphäre und interstellarem Medium, Ph.D. thesis, University of Bonn, Germany, 1998.

Kayser, S. E., Barnes, A., and Mihalov, D. J.: The far reaches of the solar wind - Pioneer 10 and Pioneer 11 plasma results, Astrophys. J., 285, 339-346, 1984.

McComas, D. J., Barraclough, B. L., Funsten, H. O., Gosling, J. T., Santiago-Muñoz, E., Skoug, R. M., Goldstein, B. E., Neugebauer, M., Riley, P., and Balogh, A.: Solar wind observations over 'Ulysses' first full polar orbit, J. Geophys. Res., 105, 10419-10 434, 2000.

Lazarus, A. and Belcher, J.: Solar Wind 6, Colorado/USA, (Eds) Pizzo, V. J., Holzer, T. E., and Scime, E. E., pp. 533-546, 1987.

Lazarus, A. J. and McNutt, R. L.: in: “The Outer Heliosphere", COSPAR Colloquium No. 1, (Eds) Grzedzielski, S. and Page, E., Warsaw/Poland, pp. 229-240, 1990.

Linsky, J. L., Brown, A., Gayley, K., Diplas, A., Savage, B. D., Ayres, T. R., Landsman, W, Shore, S. N., and Heap, S. R.: Goddard high-resolution spectrograph observations of the local interstellar medium and the deuterium/hydrogen ratio along the line of sight toward Capella, Astrophys. J., 402, 694-709, 1993.

Linsky, J. L. and Wood. B. E: The alpha Centauri Line of Sight: D/H Ratio, Physical Properties of Local Interstellar Gas, and Measurement of Heated Hydrogen (The "Hydrogen Wall") Near the Heliopause, Astrophys. J., 463, 254-270, 1996.

Naidu, K. and Barnes. A: Motion of the heliospheric termination shock. 4: MHD effects, J. Geophys. Res., 99, 17 673-17 679, 1994.

Parker, E. N.: Interplanetary dynamical processes, Interscience Publisher, New York, USA, 1963.

Potgieter, M. S.: The modulation of galactic cosmic rays in the heliosphere: Theory and models, Space Sci. Rev., 83, 147-158, 1998.

Ratkiewicz, R., Barnes, A., Molvik, G. A., Spreiter, J. R., and Stahara, S. S.: Heliospheric termination shock motion due to fluctuations in the solar wind upstream conditions: Spherically symmetric model, J. Geophys. Res., 101, 27 483-27 498, 1996.

Scherer, K.: Variations of the heliospheric shield, in: The Outer Heliosphere: Beyond the Planets, (Eds) Scherer, K., Fichtner, H., and Marsch, E., Copernicus Gesellschaft e.V., 327-355, 2000.

Scherer, K. and Fahr, H. J.: Solar cycle variations in the outer heliosphere, Geophys. Res. Lett., 30, 2, 1045, doi:10.1029/2002GL016073, 2003.

Scherer, K. and Fahr, H. J.: Solar cycle induced variations of the outer heliosphere structures, Geophys. Res. Lett., 29, DOI: 10.1029/2002GL016073, 2002.

Scherer, K., Fichtner, H., and Stawicki, O.: The heliosphere, cosmic rays and climate, in: The Outer Heliosphere: The Next Frontiers, (Eds) Scherer, K., Fichtner, H., Fahr, H. J., Marsch, E., COSPAR Colloquia Series, Vol. 11, 493-496, 2001a.

Scherer, K., Fichtner, H., Stawicki, O., and Fahr, H. J.: Cosmogenic element production in meteoroids - the influence of longterm variation in heliospheric structure, Proc. 27th Int. Cosm. Ray Conf., Hamburg, Germany, 4031-4034, 2001 b.

Scherer, K., Fichtner, H., and Stawicki, O.: Shielded by the wind: the influence of the interstellar medium on the environment of Earth, J. Atmos. Sol.-Terrst. Phys., 64, 795-804, 2002.

Steinolfson, R. S.: Termination shock response to large-scale solar wind fluctuations, J. Geophys. Res., 99, 13 307-13 314, 1994.

Wang, C. and Belcher, J. W.: Numerical investigation of hydrodynamic instabilities of the heliopause, J. Geophys. Res., 103, 247-256, 1998.

Wang, C. and Belcher, J. W.: The heliospheric boundary response 
to large-scale solar wind fluctuations: A gasdynamic model with pickup ions, J. Geophys. Res., 104, 549-556, 1999.

Whang, Y. C. and Burlaga, L. F.: Termination shock: solar cycle variations of location and speed, J. Geophys. Res., 98, 15 221$15230,1993$.

Whang, Y. C., Lu, J. Y., and Burlaga, L. F.: The termination shock: 1979-1995, J. Geophys. Res., 104, 28 255-28 262, 1999.

Wurz, P.: Detection of energetic neutral atoms, in: The Outer Heliosphere: Beyond the Planets, (Eds) Scherer, K., Fichtner, H.,
Marsch, E., Copernicus Gesellschaft e.V., 327-355, 2000.

Zank, G. P.: Interaction of the solar wind with the local interstellar medium: a theoretical perspective, Space Sci. Rev., 89, 413-688, 1999a.

Zank, G. P.: in: SOLAR WIND Nine, CP471, (Eds) Habbal, S. R. et al., American Institute for Physics, pp. 783-786, 1999b.

Zank, G. P. and Frisch, P. C.: Consequences of the change in the galactic environment of the Sun, Astrophys. J., 518, 965-973, 1999. 